\title{
MANAGEMENT OF SOCIALLY SIGNIFICANT DISORDERS
}

\author{
Spiridonov St. \\ MBAL „Higia”, Pazardjik \\ Reviewed by: Assoc. Prof. St. Popova
}

\begin{abstract}
2. Context
Sickness and health are not unavoidable states. But they apply equally to everybody. Since birth, every person carries the potential to develop various health problems due to different inheritance, prenatal influence of various factors, and peculiarities in the social and economic status of the parents. But, in life, this probability is subject to change under the influence of a number of risk factors that may cause disorders, regardless of whether or not there is susceptibility to them.

A great number of the people of diseases with bad prognoses, such as are the socially important disorders and, above all, the disorders of circulation organs and malignant formations, are connected to the complex influence of several factors. The probability for their occurrence is increased by the combined aggression towards health caused by smoking, irrational diet, heavy emotional stress, etc. Furthermore, in such combinations, the present synergism among the factors indicated is much more dangerous than an ordinary sum total of the magnitudes of their effect separately, because they affect one another, increasing the speed and strength of their aggression towards the person's health.

It should be stressed that both health and a number of the factors affecting it, as well as most of the socially significant disorders, are subject to management and control. Even if only the management of some of them is a hopeful platform for improving the health state of the nation generally and in particular - for prophylactic of the contemporary sign aggressors on the health of the Bulgarian: cardiovascular disorders, malignant formations, diabetes, chronic obstructive pulmonary disease, etc.

That is why the application of management interventions for 'arresting' risk factors provoking the onset of some socially significant disorders that have proven their efficiency are significantly intervention of their management in favor of health and life.
\end{abstract}

${ }^{22}$. Management of risk factors responsible for the increase of intensity and severity of the socially significant disorders

\section{Genetic factors}

\section{Address for correspondence:}

St. Spiridonov, MBAL "Higia", Sv. Ivan Rilski str. 3,

4400 Pazardjik

e-mail: office@higia-bg.com
Inheritance determines the main biological characteristic that forms the basis of the increase in the risk for some socially significant disorders. It matters for the predisposition for some psychiatric disorders and widely spread publicly significant chronic disorders, including the diseases of the circulation organs, diabetes, malignant formations, and others.

In reality, the disorder is a result of interaction between the genetic charge of the individual and the complex of conditions of the ambience where he lives. Despite the fact that the manifestation of that interaction varies among the different disorders, the main risk factors for the onset of mass-spread chronic disorders are in the surrounding conditions and individual behavior. Even the trends for the onset of some disorders in families may partially be explained by the influence of similar factors of the environment and behavior on the members of the entire family.

\section{Environmental factors}

There is a constant increase in the proof of the great degree of dependence between the worsening health state of the nation and the effects of the chemical, physical, economical, social, information, and family factors of the environment.

The risk physical-chemical factors of the environment are:

- Pollution of air, water, and soil;

- Harmful substances in the working environment;

- Noise levels;

- Products, harmful for consummation;

- Unsecured road-transportation system, etc. In the last decades, new health risks have arisen as a natural consequence of the progress in the industrial society. The environment became a receptacle of a great variety of synthetic materials and waste products from the industrial, agricultural, transport, and energetic working processes.

Environmental factors that affect the health are also: income, living conditions, profession. It is known, that there are many reasons because of which the health risks for poor people are more and more serious than for rich people, because of the greater number of risk factors in the environment that they inhabit:

- Heavier stress situations;

- Lower education level;

- Greater unemployment or dissatisfaction with employment; 
- Insufficient income to acquire hygienic residences, quality food, and modern basic daily and cultural necessities.

Family relationships, as an important environmental component, also affect greatly the health state. Radical changes in the family can occur if one of the parents dies or there is a separation, when children leave home, when a senior comes to live with the family. Great changes in the social dynamics can result from heavy emotional stress, which are capable of causing somatic disorders and even death. On the other hand, harmonic family environment can stimulate the psychiatric and somatic health and guarantee a healthy, nurturing atmosphere where children develop normally.

\section{Behavioral factors}

Individual habits play a decisive role for the onset of a number of heavy disorders, accidents caused by violence, and road accidents.

Many of the contemporary heavy health problems are connected with such unhealthy behavioral factors such as smoking, alcohol abuse, incorrect diet, increased medications use, speed driving, overstraining when trying to achieve goals, etc.

\subsection{Smoking}

Smoking is the main preventable reason for the onset of socially significant chronic disorders and death in our country. For that reason, in recent years there have been great efforts to achieve targeted health awareness for its limitation with still hesitant results. There is an increase of smokers in the age range 13-16 years old. Research within the framework of the European School Project (ESPAD, 2003) shows that among the students between IX and XII grades, the relative share of smokers among boys is $72.2 \%$, and among the girls, it is $77.3 \%$.

The data about smoking among professional groups called on to give personal example in the struggle with that evil show the following:

- Among school personnel: $42.9 \%$ of the surveyed answer, that they either used to smoke or are currently (as of 2002) smoking in the schools;

- Among the medical professionals in the country $-33.9 \%$ of the surveyed answer (as per 2005) that they smoke regularly.

Research in the USA shows that the mortality among smokers is $70 \%$ higher than that among non-smokers, and that smoking is a reason for 145 premature deaths per 100 thousands of the population yearly. Related to Bulgaria, that research shows that smoking is reason for about 11000 premature deaths in the country each year.

A remarkable aspect of this sad statistics is that the harm that it illustrates is entirely preventable, since the choice whether to smoke or not depends entirely on the individual. In the group of peoples that stubbornly continue to underestimate the harm from smoking, Bulgaria takes one of the leading positions. The tobacco aggression in our country is characterized by the following specifics:

- Stable positions among men;

- Expanding its hold among women over 20 years of age;

- Invasiveness among the growing up and young people of both genders in the age group up to 19 years old.

This nightmare image is not a product of pessimism. It truthfully reflects the facts of our daily life: in our home, in our work place, on the street, at the places for rest and relaxation. It is a product of strong social influences, most of which are rooted in the defective upbringing in early childhood and school years and insufficient awareness of the health worrying because of lack of knowledge about the early precursors to the disorders that smoking is the main risk factor for the development of.

In relation to the above, the following conclusion becomes evident: those who cannot give up smoking on their own must be offered a wide access to organized health structures, practicing methods and means for its stopping (treatment). However, those who are not capable or willing to stop smoking should use for smoking mixtures with low content of tars and nicotine, to not inhale the smoke deeply, to only smoke the cigarette halfway, and to decrease the number of cigarettes smoked.

\subsection{Alcohol abuse}

Alcohol abuse leads to trauma from accidents, chronic disorders, and family disruptions for thousands of Bulgarians. According to data from official statistics (National Statistical Institute), the number of people regularly using alcohol has increased for the period between 1996 and 2001 by more than 11 points. The increase is most significant among young people (15 to 24 years of age), by about 18 points.

The number of road accidents leading to heavy damages and death caused by the use of alcohol has increased". Alcohol abuse is also a cause for death by drowning and by cirrhosis and cancer of the liver. Alcohol consumption during pregnancy can cause heavy anomalies of the fetus or cause mental retardation in the development of the child. The facts show that the toll in death and in damaging human health that the alcohol takes is not limited to those who have a drinking problem. For example, road accidents are frequently caused by people who only drink occasionally, but at that point in time lost control of themselves.

In the last 20-30 years, the leaping increase in the alcohol consumption in the country lead to increase in the number of people for whom drinking is a problem, of the alcoholics, and of the severe alcoholic intoxications. The reasons for that are many, but the most important are resulting from:

- Weak-willed people mistakenly looking for an escape from a social or psycho-emotional stress they're undergoing;

- Insufficient anti-alcohol education and culture; 
- Insufficient control over the production and sale of alcohol drinks, some of which turn out to have poisonous constituents (methanol).

The health and social-economical burden connected to the alcohol invasion in our country is huge. Not only the drinkers are affected, their families' members are, too. It is a reason for increase not only in the road, but also labor and household accidents, crimes with murder and with domestic and sexual abuse, fires, and expenses for medical care. Besides causing dependency, alcohol also causes various disorders and pathological states, including behavioral and psychological deviations. A causal connection has been established between the use of alcohol and more than 60 kinds of diseases and disorders. For the majority of them, alcohol turns out to be a risk factor proportional to the quantity in which it is consumed. Alcohol consumption is connected also with other factors of risk to health: smoking, high cholesterol, arterial hypertension, obesity. The European report from 2006 "Alcohol in Europe" determines alcohol as the third most serious among the 26 factors for worsening health within the EU, after smoking and arterial hypertension.

It is important for people to understand the dangers (especially for pregnant women) deriving from the abuse of alcohol and that the people who have drinking problems or their families can find efficient help, both with their general physicians and with public organizations, working on programs directed to the limiting of alcohol abuse.

\subsection{Drugs abuse}

According to data from research made by the National Focus Center, around 315 to 330 thousands of Bulgarians between the ages of 15 and 60 have at least in their lives used a narcotic substance. It is indicative that this use is twice as large (as a relative share) among young people than among the general population.

The most frequently used narcotic substance is marijuana, followed by the group of synthetic stimulants. Based on data from research for the report of the Ministry of Health for the state of health of the citizens 2005-2007, a profile of people with at least one use of a narcotic substance in their lives was made, and it was so formulated:

- More men than women;

- Almost completely among the young;

- More likely to be well financially;

- More likely to live in larger cities.

Longitudinal observations show that in Bulgaria heroin is the substance that's most connected with problematic harmful use of narcotics. The total estimate for the number of people problematically use heroin in the country is $20-30$ thousand people, for Sofia alone - around 12 thousand.

The abuse of narcotic substances leads to heavy consequences. They harm:

\section{- The single individual, decreasing} productivity, worsening quality of life and bringing risk of premature death because of infections (hepatitis B and C and AIDS) or

overdosing;

- The family, decreasing the living standard, creating possibility of chronic stress and breakdown of family relationships and connections;

- The community, leading to increase in crime and threat to the intellectual potential and genetic pool of the nation.

All of this necessitates that the activities to improve the preventive-informational work on the problems related to harmful use of narcotic substances should be large-scale, constant, and targeted primarily towards young people (pupils and students) and parents with active help from the educational institutions, the mass communication media, writers, religious sects ministers, etc.

\subsection{Unsafe sexual behavior}

The unsafe sexual behavior is one of the social factors, negatively affecting sexual and reproductive health, and therefore the overall well-being of people. Observations on the behavior of young people show an increase of unsafe sexual practices. Indicators for that are: increased sexual activity at a young age, the high percentage of sexual contacts with several partners, and frequent change of partners. The share of young people always using condoms is increasing. However, the share of youths who do not use condoms at all is still high. The results of this behavior are: on the one hand - unplanned pregnancy at a young age, voluntary abortions or early marriages and births, and on the other sexual transmitted infections, hepatitis $\mathrm{B}$ and $\mathrm{C}$, HIV/AIDS.

\subsection{Irrational and unbalanced eating.}

It takes an important place in the complex of risk health factors. It is well-known that Bulgaria is a country where high-calorie food is consumed. A basic share in the consumption structure here belongs to bread and animal fats. The consumption (in the winter and spring in particular) of vitamins and microelements-rich vegetables and fruits is low. The use of cooking salt is traditionally high, which probably has a causal connection to the high incidence of arterial hypertension and the high mortality caused by circulation organs disorders here.

It is important to note the fact, that recently there are trends towards lower-protein diets among older people, which, if it continues, might provoke the appearance of a number of nutritional shortages conditions.

Looked at through this prism, it can be claimed that the contemporary dietary problems of the Bulgarian people are connected more with an imbalance among the types of foods consumed, rather than insufficient nutrition.

One of the essential problems in this case is the developing obesity across all age groups, where it is most acute in the age range between 45 and 60 years old.

The second important problem is the connection between irrational eating and the onset of some disorders of the blood circulation organs. 
There are many arguments regarding the role of food rich in animal fats in the increase of cholesterol in the blood and the decrease of that of the high-density lipoproteins. It has been proved, that the mentioned deviations in these two factors are related to atherosclerosis and other cardiovascular system disorders. It has been established, that people who consume food lean on animal fats are less likely to experience a cardiac infarct. In the countries with low coronary diseases, not only the adults, but also children have lower levels of cholesterol than many Bulgarian children. Salt is also a risk factor for health. Research shows that one of the basic causes for the widely spread in Bulgaria incidence of arterial hypertension is the high content of cooking salt in the consumed food. This high incidence and its part as number one risk factor for the appearance of cardiac infarcts and brain strokes require that Bulgarians should limit their intake of salt by:

- Cooking with smaller amounts of salt;

- Removing salt cellars from the table;

- Avoiding overly salty meat products: slanina/bacon, pastarma, sizdirma;

- Paying attention to the information on the packaged food labels and their salt contents.

The third important problem is the connection of some food and nutritional supplements with the development of cancer diseases. It has been proven that the presence or absence of some components in the food products, as well as some methods for preparing food, might have a relation to the incidence of malignant formations. Such include:

- Substances for improving the color or taste of foods, prohibited by the health organs;

- Molds in food products, which release the cancerogenic factor alpha toxin;

- Foods poor in fibrous substances (cellulose) and rich in fats;

- Marinated, as well as coal-roasted/baked foods.

\subsection{Decreased physical activity}

The decreased physical activity is a way of life for more than two generations of Bulgarians. A significant part of our compatriots drive cars and use other vehicles when going to work. The work itself, for most of the work force, is connected with small or no physical activity. Even for their entertainment, people are more frequently spectators than participants. The relative lack of physical activity in the nation leads to the decrease in strength indicators, possibilities to adapt, and sports form, among the young as well as the aging.

In recent years, there has been a hopeful return of interest to physical exercises and sports form. There are many young people now who engage in mountain tourism, skiing, biking, swimming, aerobics, and other forms of physical activity.

Despite the increasing numbers of people engaging in physical exercises, in most of their cases it is not systematic or sufficiently energetic for achieving the health-optimum benefit. The frequency of participation is higher among men than among women; higher among the younger than the older; higher among the more educated than among the less so; higher among the richer than among the poor. It is important to note that:

- In school sports, there is too much attention focused on group sports, where the participant is not always active;

- Regarding those working in business, exercising programs are an exception company managements underestimate the role of those programs for the strengthening and protecting the health of their employees and in the increase of their labor's productivity;

- Most of the older people do not exercise, which leads to atrophy of their muscle tissue with negative results on the correct body position, necessary for the effective motion muscle strength, mobility of their joints, the cardiac-motor and respiratory endurance;

- "Medical specialist do not do what is necessary for the active stimulation of their patients towards physical exercises, and when those are recommended, they are not with a powerful message, due to which the benefit from them is limited;

- On a national level, the former National Agency for Youth and Sports of the Council of Ministers did not show the necessary activity for the development of informational initiatives, school, university, and worker programs and targeted projects for the increase of participation of Bulgarian citizens in sports activities.

From what has been mentioned so far regarding the behavioral factors, the conclusion must be drawn that the Bulgarian citizen, should he wish to not fall sick, disabled, or prematurely dead, should undertake such a lifestyle which enables him to limit, as much as possible, the risk of the health-damaging personal habits. That will be an expression of high culture, self-respect, responsibility towards his family and community, and, not in the last place, of economic reasonability.

One of the benefits of the civic society is that the people it consists of choose their ways of life on their own. But from the position of managing socially significant diseases, this choice must not be destructive, especially as regards the national priorities, an important part of which takes the people's health. In that respect, the role and responsibility of the media of mass communication for the formation in each Bulgarian a behavior, relative to the healthy way of life, increases.

The presence of risk factors does not necessarily mean that the development of a disorder is unavoidable. But due to the cumulative effect of additional factors, a downturn in the state of health may occur. The chain of occurrences, negatively affecting health, can be short, such as in road ac- 
cidents, or long and complicated, such as the onset of coronary disease and following infarct.

The risk of disorder may sometimes be singular, such as not undergoing immunization against a disease. In other cases, it's a set of factors that at work. For example, the factors indicating the occurrence of disorders of the circulatory system organs can be: inheritance, irrational eating, smoking, overeighing, untreated arterial hypertension, insufficient physical activity, psycho-emotional stress, and probably another number of unknown factors of social, abiotic, and biotic nature.

Since now and in the near future, the financial resources for healthcare in Bulgaria (as compared to the developed EU states) will be limited, it is vital that people actively participate in the activities aimed at limiting the risk if disorders and trauma which can be prevented.

This, however, does not mean that the responsibility for the state of the individual health and control over it is entirely the individual's. For example, it is known that the social-economic factors are powerful determinants for the health status and that people can only exercise partial control over them. Also, it is outside the potential of the individual to lower the action of a number of health-damaging environment factors. The role of the individual in the struggle to modify that environment is to participate as a citizen in the stress activities in the key links of the system and processes. But as regards the control over risk factors, individuals must comply with the formally conducted events as run by the national and municipal organs.

And, finally, despite the fact that people are able to decrease the risk of disease and accidents by change in their behavior, the effects on their health will be registered over a large period of time. When a person knows that it is not healthy to consume over much of calories and fats, he can self-impose some limits. Also, he must know that the use of cigarettes, alcohol, and drugs could cause addiction to those substances.

It is erroneous to claim that the behavioral forms of the individual completely depend on him. Even knowing the harmful effect of the risk factors sometimes makes the effort of people aimed at decreasing their harmful effect harder.

The health behavior, including the effective use of the institutional network of centers for prevention and control of public health is a basic area, where the personal responsibility of the citizen towards the individual, family, and community health increases.

\section{SOURCES:}

1. Health for all $21^{\text {st }}$ century, Basic policies for reaching health for all in the European region. WHO - European regional bureau, Copenhagen.

2. "Healthcare" - statistical edition 2006 and 2008. National Statistical Institute/ National Center for Health Information

3. National health strategy "Better health for better future for Bulgaria", 2001.

4. National health strategy - $2008-2013$.

5. Improving healthcare in Bulgaria: variants for reforms. Report of the World Bank. 2003.

6. State of health of the citizens $2005-2007$, primary investment in the future of the nation. Report of the Ministry of Health 2009.

7. Shipkovenska, E. Health of the nation - work of all and everybody. Magazine "Health Management", issue 41, 2004. 